\title{
An Alternative Strategy for Photon-Pair Generation using Integrated Photonics on Silicon Wafers (1640968-Y4)
}

\author{
SHAYAN MOOKHERJEA \\ Department of Electrical and Computer Engineering, University of California, San Diego, MC 0407 La \\ Jolla CA 92093-0407 USA \\ Email:smookher@ucsd.edu
}

Summary of a Project Outcomes report of research funded by the U.S. National Science Foundation under Project Number 1640968 (Year 4).

\section{Introduction and Goals}

The overall goals of this project to investigate and develop integrated photonpair generation and other types of quantum optics devices that are needed for scalable implementation of measurement-device-independent quantum key distribution (MDI-QKD) and similar quantum communications protocols. The UCSD sub-project focused on the development of key building blocks for microchips in both silicon photonics and lithium niobate photonics which are cost-effective, leverage modern micro-fabrication platforms, and improve scalability.

\section{Activities}

Previously, our research activities in this project focused on developing pair sources using integrated silicon photonics technology. The spontaneous fourwave mixing (SWFM) nonlinearity in silicon nanophotonic waveguides is reasonably strong $\left(\gamma=200 \mathrm{~W}^{-1} \mathrm{~m}^{-1}\right)$ but meter-length waveguides cannot be fabricated on silicon chips, because of the typical loss (typically $\alpha=1 \mathrm{~dB} \cdot \mathrm{cm}^{-}$ $\left.{ }^{1}\right)$ which limits the effective length $\left[\mathrm{L}_{\mathrm{eff}}=\left(1-\mathrm{e}^{-\alpha \mathrm{L}}\right) / \alpha\right]$ to a few centimeters, at most. Also, the pump power is limited to much less than a Watt, since nonlinear losses from two-photon absorption and free-carrier generation at higher pump powers limit both the available pump power and the number of photon pairs that can be extracted from the end of the waveguide. Compared to a simple waveguide, a waveguide-coupled microring resonator is a useful and compact device which can generate several million photon pairs per second from milliwatt level pump powers. For this purpose, we have used both single microring resonators as well as the silicon coupled-resonator optical waveguide structure[1]-[4], which has a resonantly enhanced four-wave mixing nonlinearity[5]. We have demonstrated the time-energy entangled nature of the photon pairs generated by our devices around $1550 \mathrm{~nm}[6]$. However, such devices are sensitive to fabrication disorder[7] and in practice, an optimized single microring resonator may achieve better performance (or is at least easier to tune). These demonstrations benefit from improvements we have made in monitoring and stabilizing the pair generation process[8]. 
Activities performed this year include the design, fabrication and poling of a spontaneous parametric down-conversion (SPDC) waveguide in periodicallypoled thin-film lithium niobate SPDC device, and measurements of photon-pair generation in it. Lithium niobate (LN) is a well-known crystal in nonlinear and quantum optics, and used in photon-pair sources, electro-optic modulators and wavelength converters, because it has a wide transparency window, as well as a large second-order nonlinear coefficient. In recent years, LN on insulator wafers, or LNOI have become increasingly popular. In cross section, LNOI consists of a top crystalline thin-film lithium niobate layer, which can be bonded to a silicon handle wafer with an insulator layer (oxide) in-between. Such wafers can be used to selectively incorporate LN with other components of a silicon photonics wafer fabrication process, through bonding and handle removal.

In the SPDC process, two photons are generated under the energy and momentum conservation condition. Because of the material and waveguide dispersion, the phase matching condition cannot be realized naturally for all waves polarized along the TE axis. But quasi-phase matching can be achieved in a ferroelectric material, including lithium niobate, wherein the concept is to compensate the phase mismatch by periodically reversing the sign of second order nonlinear coefficient of the nonlinear material, which allows the generated power (number of generated photons) to grow monotonically. For the waveguide we used, the poling period is about 2.8 microns, which is one order of magnitude shorter than the typical poling period used in conventional periodically-poled lithium niobate (PPLN) waveguides. Figure 1 shows an image of a small microchip containing more than twenty periodically-poled thin-film LN waveguides, developed during this project.

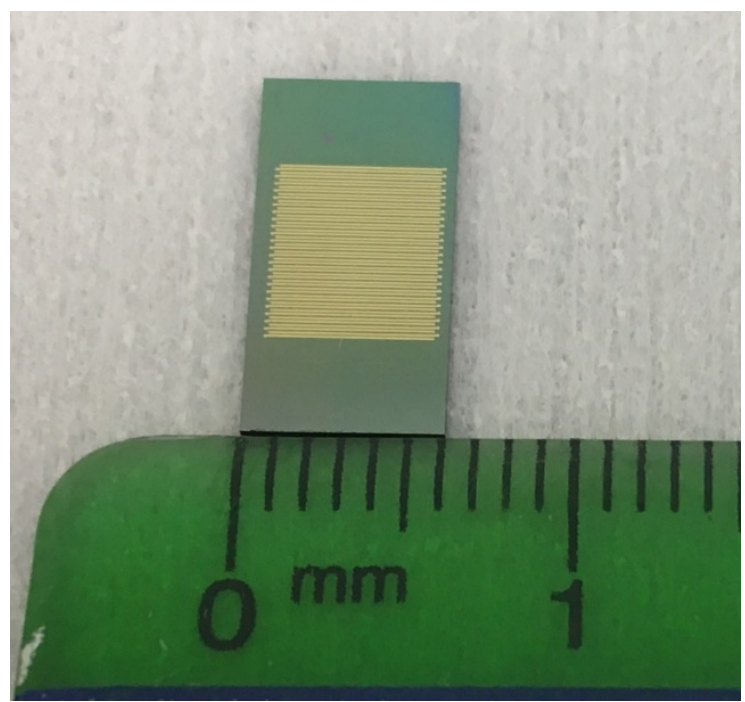

Figure 1 Image of a fabricated chip containing twenty four periodically-poled thin-film lithium niobate shallow-etched waveguides with varying waveguide widths. 
Figure 2 shows a nonlinear microscope image of a section of a poled waveguide, demonstrating that a near-ideal $50 \%$ duty cycle was achieved in the poling period. We started with a $300 \mathrm{~nm}$ thick X-cut LNOI chip, this chip was then periodically poled and etched into waveguides. We consider SPDC process from about $780 \mathrm{~nm}$ to $1560 \mathrm{~nm}$, using a shallow-etched ridge waveguide, with the etching depth being $50 \mathrm{~nm}$. The optimal waveguide width was defined by maximizing the conversion efficiency from the second harmonic generation process, which is the reverse process of SPDC. The target waveguide width is about $1.2 \mathrm{um}$. The periodically poled domains should be uniform at least to a few microns on either side of the waveguide center axis. To evaluate the poling quality, we developed a non-destructive nonlinear microscopy technique, that allows us to image the inverted domains and optimize our poling recipe. The relatively bright rectangle in the middle of the image represents the etched waveguide. The black lines that start from the positive electrode and end at the ground electrode are the domain walls. The poled and unpoled region are separated by the domain walls. Similar images were generated over multiple segments of the waveguide to verify the poling quality and optimize the poling recipe.

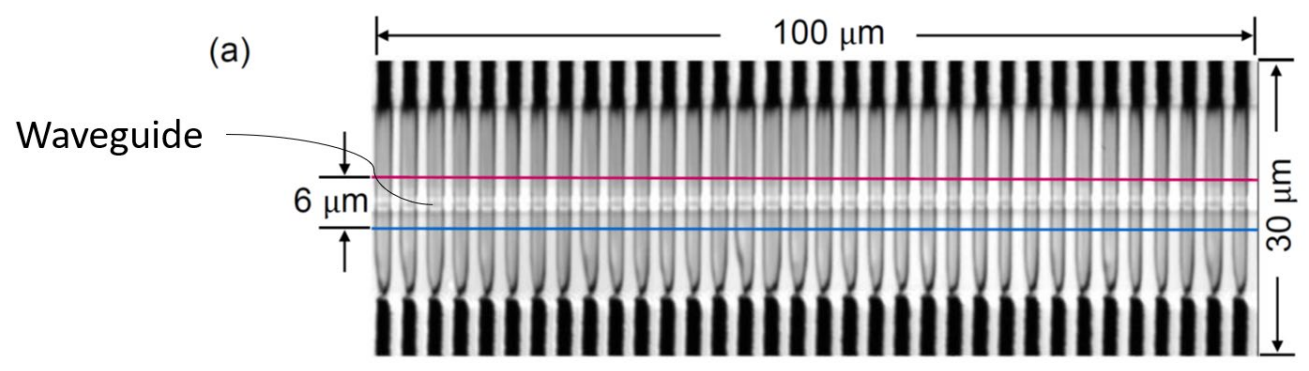

(b)

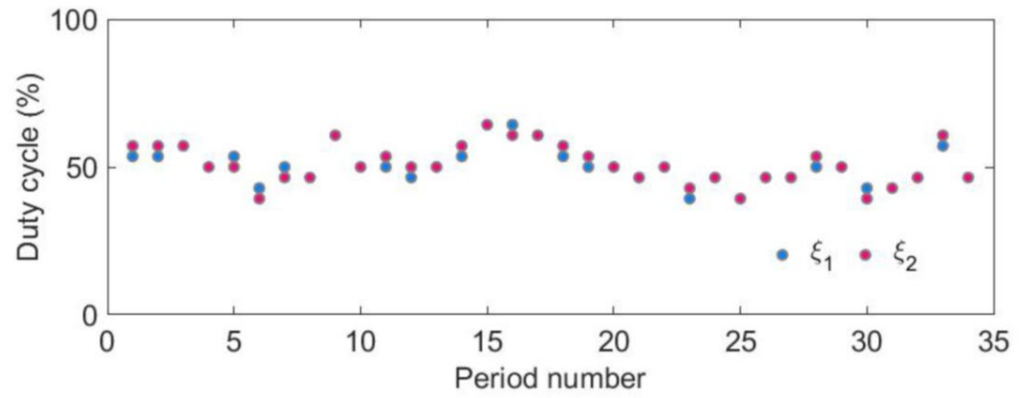

Figure 2 (a) Scanning second-harmonic microscopy image, showing poled domains. Two line scans near the waveguide, separated by 6 um, are studied. (b) Image processing was performed on line scans to determine the locally-varying poling duty cycle (ideal $=50 \%$ )

\section{Impact}

The waveguide is pumped by a laser diode at the wavelength of around $785 \mathrm{~nm}$. The PPLN chip is mounted on a temperature controlled stage for tuning and 
stabilization. The output light was filtered to reject the pump, and select the signal and idler photons. The filter assembly is followed by a $50 \%-50 \%$ splitter. Photons were detected by two SNSPD detectors. The SPDC generation rate (on chip) was $4.5 \times 10^{7}$ pairs $\mathrm{s}^{-1} \mathrm{~mW}^{-1}$ and the brightness was $\mathrm{B}=5.6 \times 10^{7}$ pairs $\mathrm{s}^{-}$ ${ }^{1} \mathrm{~nm}^{-1} \mathrm{~mW}^{-1}$. We measured a CAR of about 1,600 at $4 \times 10^{6}$ pairs s$^{-1}$ (on-chip). We also measured a heralded second-order autocorrelation $\mathrm{g}^{(2)}(0) \sim 0.022$ at 7 x $10^{5}$ pairs $\mathrm{s}^{-1}$ which demonstrates strong anti-bunching of heralded single photons. Approximately one order of magnitude improvement was observed and reported in the figure-of-merit (PGR x CAR) over silicon microrings. An experiment showed two-photon time-energy entanglement visibilities exceeding $99 \%$ without subtraction of accidentals (see Fig. 3). Improving the generation rate at the milliwatt scale pump power levels may make sources easier to use and deploy, since $1550 \mathrm{~nm}$ wavelength, milliwatt-level laser diodes are inexpensive, compact and energy efficient, especially when compared to traditional Ti:Sapphire lasers that are used to pump traditional lithium niobate crystals for pair generation.

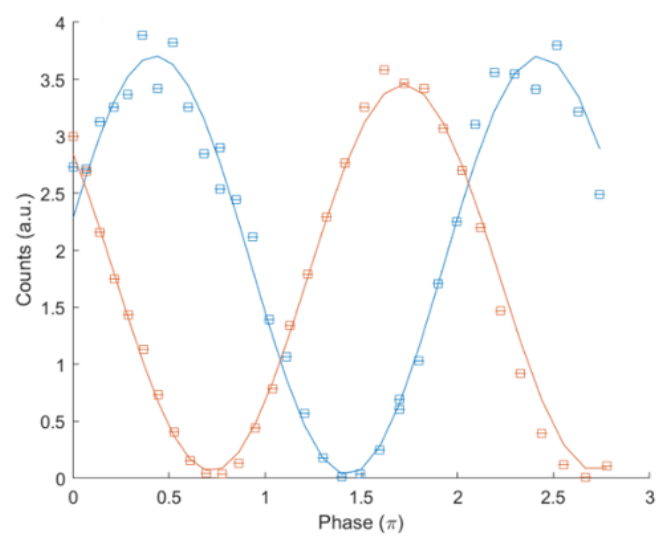

\begin{tabular}{|c|c|}
\hline \multicolumn{2}{|l|}{ Blues: } \\
\hline Visibility(raw): & $99.3+/-1.9 \%$ \\
\hline Visibility(fit): & $98.4 \%$ \\
\hline Oranges: & \\
\hline Visibility(raw): & $99.5+/-1.8 \%$ \\
\hline Visibility(fit): & $96.4 \%$ \\
\hline
\end{tabular}

Figure 3 Summary of measurements of time-energy entanglement of the generated photon pairs at $1560 \mathrm{~nm}$ using a Franson interferometer. A high visibility of about $99 \%$ was measured.

One of the advantages of the integrated device is that the coupling to the resonator is stable and can be controlled, although, fundamentally, the dispersion (i.e., wavelength sensitivity) of the directional coupler can be large 
because of the high index contrast between the waveguide core and cladding materials. However, the same large dispersion issue also affects the coupledresonator device and can sometimes be used for added functionality[9], [10].

The work of three graduate students at UCSD was supported by this project. They received hands-on training in the fields of electrical engineering, physics, device fabrication and measurement and materials science. Researchers and Students interacted with government laboratory researchers (including NIST, JPL, Sandia). Results have been published in peer-reviewed journals and presented at internationally recognized conferences, as well as workshops, seminars, and meetings. One of our graduate students completed a multi-month internship at a major semiconductor company. We hosted high-school students for a sequence of demonstration experiments in optics and photonics (see Fig. 4).

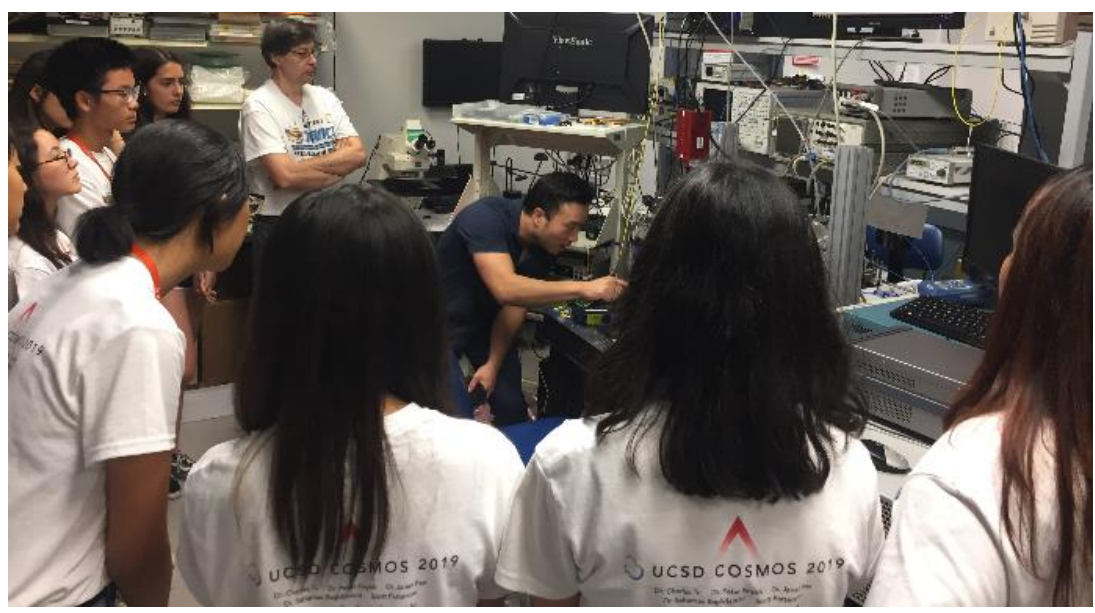

Figure 4 Outreach program to "COSMOS" high school students by a graduate student in our lab.

\section{Potential Subsequent Extensions}

More research is needed in reducing chip-to-fiber coupling losses, which currently limit the heralding efficiency to about 5\%. If the laser is integrated with the microchip, then the edge coupling losses are not as relevant. However, this is at an early stage of development and has not yet been realized.

One technical challenge is that short pump pulses are usually used for pair generation (from mode locked lasers), and although integrated electro-optic modulators can be used to carve pump pulses, they may need a very wide bandwidth. However, we have shown how such modulators can be integrated with other types of photonics components on a photonic microchip which leverages foundry fabrication methods[11]. 
A complete ecosystem of integrated lithium niobate photonics is also at an early stage of development, and does not yet have "library" components such as filters, multiplexers and couplers which are already available in silicon photonics.

A fundamental challenge of high index contrast integrated photonics using microresonators is the sensitivity to disorder. This is particularly true for the coupled-resonator configuration[12], [13] but is also relevant for waveguides, single microrings, and periodically poled structures since the group velocity dispersion plays a role in the pair generation process in each of these structures. However, we have also studied ways to study and potentially compensate for disorder after fabrication [14]-[16] and dispersion compensation is not yet the limiting factor in performance (compared to loss improvements and improving fabrication yield).

The silicon photonic devices which we studied use the efficient all-optical fourwave mixing (FWM) nonlinearity that occurs in a highly confined waveguide (cross sectional mode area about 0.2 squared microns). Physics theory suggests that there are other interesting Kerr nonlinear effects that might occur in appropriately dispersion-engineered devices, such as the generation of bright and dark solitons[17], [18] which result in the high confinement of light at elevated power levels. An experimental study may benefit from using materials in which the nonlinear losses (such as two-photon absorption) are lower than in silicon or III-V semiconductors. Soliton generation is an important part of the process of forming frequency combs in silicon nitride microresonators.

\section{Open-Access Reporting Initiative}

PRAISE: This open-access document is provided in support of our PRAISE

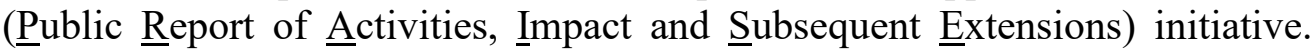
What is it? An open-access document shared with the public which describes the research outcomes of publicly-funded projects such as those funded by the U.S. NSF (National Science Foundation).

\section{References}

[1] S. Mookherjea, "Spectral characteristics of coupled resonators," J. Opt. Soc. Am. B, vol. 23, no. 6, p. 1137, Jun. 2006, doi: 10.1364/JOSAB.23.001137.

[2] S. Mookherjea, D. S. Cohen, and A. Yariv, "Nonlinear dispersion in a coupled-resonator optical waveguide," Opt. Lett., vol. 27, no. 11, p. 933, Jun. 2002, doi: 10.1364/OL.27.000933. 
[3] J. R. Ong and S. Mookherjea, "Quantum light generation on a silicon chip using waveguides and resonators," Opt. Express, vol. 21, no. 4, p. 5171, Feb. 2013, doi: 10.1364/OE.21.005171.

[4] M. L. Cooper et al., "235-ring Coupled-Resonator Optical Waveguides," in Conference on Lasers and Electro-Optics 2010, San Jose, California, 2010, p. CTuHH3. doi: 10.1364/CLEO.2010.CTuHH3.

[5] J. R. Ong, R. Kumar, and S. Mookherjea, "Silicon microring-based wavelength converter with integrated pump and signal suppression," Opt. Lett., vol. 39, no. 15, p. 4439, Aug. 2014, doi: 10.1364/OL.39.004439.

[6] R. Kumar, M. Savanier, J. R. Ong, and S. Mookherjea, "Entanglement measurement of a coupled silicon microring photon pair source," Opt. Express, vol. 23, no. 15, p. 19318, Jul. 2015, doi: 10.1364/OE.23.019318.

[7] S. Mookherjea, J. R. Ong, X. Luo, and L. Guo-Qiang, "Electronic control of optical Anderson localization modes," Nature Nanotech, vol. 9, no. 5, pp. 365-371, May 2014, doi: 10.1038/nnano.2014.53.

[8] M. Savanier, R. Kumar, and S. Mookherjea, "Optimizing photon-pair generation electronically using a $p-i-n$ diode incorporated in a silicon microring resonator," Appl. Phys. Lett., vol. 107, no. 13, p. 131101, Sep. 2015, doi: 10.1063/1.4932047.

[9] S. Mookherjea, "Using gain to tune the dispersion relation of coupledresonator optical waveguides," IEEE Photon. Technol. Lett., vol. 18, no. 5, pp. 715-717, Mar. 2006, doi: 10.1109/LPT.2006.871144.

[10] S. Mookherjea, "Semiconductor coupled-resonator optical waveguide laser," Appl. Phys. Lett., vol. 84, no. 17, pp. 3265-3267, Apr. 2004, doi: 10.1063/1.1719278.

[11] X. Wang, P. O. Weigel, J. Zhao, M. Ruesing, and S. Mookherjea, "Achieving beyond-100-GHz large-signal modulation bandwidth in hybrid silicon photonics Mach Zehnder modulators using thin film lithium niobate," APL Photonics, vol. 4, no. 9, p. 096101, Sep. 2019, doi: 10.1063/1.5115243.

[12] M. L. Cooper and S. Mookherjea, "Modeling of Multiband Transmission in Long Silicon Coupled-Resonator Optical Waveguides," IEEE Photon. Technol. Lett., vol. 23, no. 13, pp. 872-874, Jul. 2011, doi: 10.1109/LPT.2011.2141657.

[13] S. Mookherjea and M. A. Schneider, "Avoiding bandwidth collapse in long chains of coupled optical microresonators," Opt. Lett., vol. 36, no. 23, p. 4557, Dec. 2011, doi: 10.1364/OL.36.004557.

[14] M. L. Cooper, G. Gupta, J. S. Park, M. A. Schneider, I. B. Divliansky, and S. Mookherjea, "Quantitative infrared imaging of silicon-on-insulator microring resonators," Opt. Lett., vol. 35, no. 5, p. 784, Mar. 2010, doi: 10.1364/OL.35.000784. 
[15] S. Mookherjea and H. R. Grant, "High dynamic range microscope infrared imaging of silicon nanophotonic devices," Opt. Lett., vol. 37, no. 22, p. 4705, Nov. 2012, doi: 10.1364/OL.37.004705.

[16] Y. Shen, I. B. Divliansky, D. N. Basov, and S. Mookherjea, "Perfect set-and-forget alignment of silicon photonic resonators and interferometers," in Optical Fiber Communication Conference/National Fiber Optic Engineers Conference 2011, Los Angeles, California, 2011, p. PDPC3. doi: 10.1364/OFC.2011.PDPC3.

[17] B. Crosignani, A. Yariv, and S. Mookherjea, "Nonparaxial spatial solitons and propagation-invariant pattern solutions in optical Kerr media," Opt. Lett., vol. 29, no. 11, p. 1254, Jun. 2004, doi: 10.1364/OL.29.001254.

[18] A. Ciattoni, B. Crosignani, S. Mookherjea, and A. Yariv, "Nonparaxial dark solitons in optical Kerr media," Opt. Lett., vol. 30, no. 5, p. 516, Mar. 2005, doi: 10.1364/OL.30.000516. 